\title{
Deficits in orthographic knowledge in children poor at rapid automatized naming (RAN) tasks?
}

Article

Accepted Version

Powell, D., Stainthorp, R. and Stuart, M. (2014) Deficits in orthographic knowledge in children poor at rapid automatized naming (RAN) tasks? Scientific Studies of Reading, 18 (3). pp. 192-207. ISSN 1088-8438 doi:

https://doi.org/10.1080/10888438.2013.862249 Available at https://centaur.reading.ac.uk/35151/

It is advisable to refer to the publisher's version if you intend to cite from the work. See Guidance on citing.

To link to this article DOI: http://dx.doi.org/10.1080/10888438.2013.862249

Publisher: Routledge

All outputs in CentAUR are protected by Intellectual Property Rights law, including copyright law. Copyright and IPR is retained by the creators or other copyright holders. Terms and conditions for use of this material are defined in the End User Agreement.

www.reading.ac.uk/centaur 
Central Archive at the University of Reading

Reading's research outputs online 


\section{Deficits in Orthographic Knowledge in Children Poor at Rapid Automatized Naming (RAN) Tasks?}

Keywords: Rapid Naming; Orthographic processing; reading acquisition; phonological processing

Authors: Daisy Powell ${ }^{\mathrm{a}, 1}$, Rhona Stainthorp ${ }^{\mathrm{b}}$, and Morag Stuart ${ }^{\mathrm{c}}$

${ }^{a}$ University of Roehampton, Department of Psychology, Whitelands College, Holybourne Avenue, London SW15 4JD, UK.

${ }^{\mathrm{b}}$ Institute of Education, University of Reading, London Road Campus, 4 Redlands Road, Reading, Berks, RG1 5EX.

${ }^{\mathrm{c}}$ Institute of Education, University of London, Department of Psychology and Human Development, 20 Bedford Way, London, WC1H 0AL UK

Corresponding author: Daisy Powell, Institute of Education, University of Reading, London Road Campus, 4 Redlands Road, Reading, Berks, RG1 5EX, UK.

Email: d.a.powell@reading.ac.uk; Tel: +44 118378 2797; Fax: +44 1183788834.

${ }^{1}$ Daisy Powell is now at the Institute of Education, University of Reading. 
Orthographic Knowledge and RAN

\begin{abstract}
The degree to which orthographic knowledge accounts for the link between Rapid Automatized Naming (RAN) and reading is contested, with mixed results reported. This longitudinal study compared two groups of 10-11 year old children, a low RAN group $(\mathrm{N}=69)$ and matched controls $(\mathrm{N}=74)$ on various measures of orthographic knowledge. The low RAN group showed a deficit in orthographic knowledge, both at the level of sub-word letter sequences and of whole words, as well as an unexpected strength in orthographic learning. Our findings underline the persistence of RAN-related reading problems, and raise questions about reading strategies in this group.
\end{abstract}




\section{Deficits in Orthographic Knowledge in Children Poor at Rapid Automatized Naming (RAN) Tasks?}

The association between performance on Rapid Automatized Naming (RAN) tasks and progress learning to read, first noted by Denckla and Rudel (1974), is now well established. RAN tasks require speeded naming of arrays of familiar items (letters, digits, objects, or colours). RAN is one of the most powerful predictors, alongside phonological awareness (PA), of children's word reading across a wide range of languages (see Norton \& Wolf, 2011, for a review). Like reading, RAN tasks are complex and multi-componential, requiring coordinated input from diverse cognitive and perceptual processes. Given the multi-faceted nature of RAN, theorists have struggled to find a simple explanation for its relationship with reading, which remains a contentious issue.

A traditional view of the RAN-reading link emphasizes the phonological component of the RAN task seeing RAN as an index of speed of access to phonological information(Vellutino, Fletcher, Snowling, \& Scanlon, 2004; Wagner et al., 1997). Others (e.g. Wolf, Bowers, \& Biddle, 2000) propose that RAN tasks index processes that are in part independent of phonology. In support of this, several studies have demonstrated that RAN and PA each account for unique variance in reading (e.g. Badian, 1993; Manis, Seidenberg, \& Doi, 1999; Powell, Stainthorp, Stuart, Quinlan \& Garwood, 2007), and that some poor readers have RAN deficits in the absence of PA deficits (Powell et al., 2007; Wolf \& Bowers, 1999).

The dissociation between PA and RAN raises questions about which nonphonological cognitive factors might underlie the RAN-reading link. Several candidates have been suggested, including speed of processing (Kail, Hall, \& Caskey, 1999), visual attention span (Bosse, Tainturier, \& Valdois, 2007), visual processing (Stainthorp, Powell, 
Stuart, Quinlan \& Garwood, 2010), serial processing (Georgiou, Parrila, Cui, \& Papadopoulos, 2013), and higher level cognitive control (Protopapas, Altani, \& Georgiou, 2013), while it was Bowers and Wolf (1993) who first suggested a mechanism whereby cognitive factors underlying poor RAN performance could lead to reading deficits by impairing orthographic knowledge. This could present a clear developmental impediment as reading is thought to shift from a reliance on decoding to orthographic processes as the reading system matures (Ehri, 2005). Bowers and Wolf proposed that if visual identification of letters is too slow to allow for the simultaneous activation of sequences of letter representations when reading, children would struggle to store knowledge of commonly occurring orthographic patterns. Supportive evidence was provided by Bowers, Sunseth and Golden (1999), who reported relatively poor performance on a "quick spell task" requiring children to recall letters in briefly presented letterstrings, in a low RAN group.

However, while some researchers (Georgiou, Parrila, \& Kirby, 2009; Manis et al., 1999) also reported that RAN is more strongly linked to orthographic than phonological tasks, others failed to find a clear link between orthographic knowledge and RAN (e.g. (Bowey \& Miller, 2007; Conrad \& Levy, 2006; Moll, Fussenegger, Willburger, \& Landerl, 2009; Torgesen, Wagner, Rashotte, Burgess, \& Hecht, 1997). Indeed, van den Boer, de Jong, and Haentjens-van Meeteren (2013) recently found, like Moll et al. (2009), that the effect of RAN was as strong for pseudowords as it was for words, which they argue speaks against a specific link between RAN and orthographic knowledge.

As highlighted by Apel (2011), there are ambiguities about what is meant by orthographic knowledge, and how best to conceptualize its role in reading. The term orthographic knowledge is often used to describe two rather different sorts of knowledge, which have sometimes been explicitly contrasted in the literature (e.g., Bowers et al., 1999; 
Georgiou et al., 2009; Powell, Stainthorp \& Stuart, 2008). The term can refer to word-level knowledge of individual words' spellings, necessary for reading irregular words, like 'yacht', which are frequent in English and cannot be decoded solely according to grapheme-phoneme correspondence rules. It can also refer to sub-word level orthographic knowledge described by Apel (2011) as "orthographic pattern learning": accumulated statistical knowledge about how letters typically combine in words. If RAN is differentially related to whole-word and sub-word level orthographic knowledge, then the fact that different studies have assessed different aspects of orthographic knowledge may contribute to the mixed findings reported.

To develop a full understanding of RAN's putative link to orthographic knowledge, it is necessary to first extricate the role of RAN from other known correlates of orthographic knowledge, namely phonological processing and experience with print. Share's (1995) selfteaching hypothesis sees phonological recoding, of which PA is a core component, as the mechanism for orthographic learning. On the other hand, Cunningham and Stanovich (1993) demonstrated that experience with print accounted for unique variance in orthographic skill, independent of phonological skills. To our knowledge, print exposure has not previously been assessed explicitly in studies relating RAN to orthographic knowledge, so it remains possible that any such link may be spurious, driven by the relationship between print exposure and both RAN and orthographic knowledge.

The current research had two main aims. Previous studies provide conflicting data with reference to the persistence of RAN difficulties and associated poor reading, with some (Wagner et al., 1997; Torgesen et al., 1997) suggesting that, in English at least, the RANreading link decreases with age, and others (Kirby, Parrila, \& Pfeiffer, 2003; Landerl \& Wimmer, 2008) finding no diminution over time. Our longitudinal study offered a further opportunity to assess the persistence of RAN deficits and associated reading problems, in a 
group of English children with RAN difficulties relative to a control group matched on PA and other key cognitive factors. We assessed whether, nearly three years after they were first tested, the low RAN group were still slower on the RAN task and worse readers than controls.

The second aim of the current research was to examine orthographic skills in the low RAN group, relative to matched controls, in response to mixed findings previously reported. To do this we carried out a range of assessments of orthographic knowledge. Our reading measure yielded separate scores for regular word, irregular word and pseudoword reading. Irregular word reading (e.g. pint) is thought to be a stronger test of orthographic knowledge as, unlike regular words (e.g. mint) and pseudowords (e.g. sint), irregular words cannot be decoded using phonological recoding skills alone. Pseudoword reading, on the other hand, provides a purer measure of online decoding skills. Thus, a selective difficulty with irregular words in the low RAN group would suggest deficient orthographic knowledge. We recorded word reading speed as well as accuracy data because RAN appears to be more strongly linked to reading fluency than reading accuracy, certainly in more consistent orthographies than English (e.g. Georgiou, Torppa, Manolitsis, Lyytinen, \& Parrila, 2010). In addition, we aimed to target different aspects of orthographic knowledge, using tests of both word-level orthographic knowledge and sub-word level orthographic patterns, to examine whether either or both aspects are implicated in RAN-related reading difficulties. We also investigated the children's ability to store new orthographic knowledge.

Our final aim was to address the question of whether the low RAN group's orthographic knowledge might be a consequence, rather than a cause of their poorer reading. To that end we assessed print exposure, which has not typically been assessed in studies investigating links between RAN and orthographic knowledge, certainly where the 
additional, confounding influence of phonological awareness is controlled, as in the current research.

As noted above, isolating an independent role for RAN in orthographic knowledge is challenging, given the competing influences of phonological skills and print exposure. Our research adds to the literature in this field through simultaneously addressing both potential confounds in a detailed exploration both of word and sub-word level orthographic knowledge, and of orthographic learning. First, our experimental design provides a control for the influence of phonology, as the low RAN and control groups were carefully matched on PA, as well as other key variables. Second, we accounted for the role of print exposure. Finally, the current research addressed the issue of whether RAN-related reading difficulties persist beyond the early stages of literacy learning, a question with important implications at this point of transition between primary and secondary school, where progress will increasingly depend on independent reading skills.

\section{Methods}

\section{Participants}

Data reported here were obtained from a sample of British school children as part of a longitudinal investigation of RAN and reading. Previous analyses of this sample are reported in Powell et al. (2007) and Stainthorp et al. (2010). Children were initially recruited when they were in Years 3 and 4 (equivalent to US $2^{\text {nd }}-3^{\text {rd }}$ Grade) of nine state-funded primary schools in south-east England to form two groups: a low RAN group ( $N=74,36$ in Year 3 and 38 in Year 4) and a control group ( $\mathrm{N}=78 ; 36$ in Year 3 and 42 in Year 4). On baseline measures, the low RAN group performed below the normal range (defined as at least one SD 
below the mean) on RAN (letters and digits), but within the normal range for PA. Controls performed within the normal range on both RAN and PA. Groups were closely matched on PA, age, phonological memory and verbal and non-verbal ability, though the low RAN group were, consistent with group allocation, significantly slower on RAN, and significantly worse word readers than controls at baseline. At baseline the low RAN group were also significantly slower on a measure of simple reaction time (SRT), so SRT was included as a covariate in RT analyses reported here. Full details of baseline measures are reported in Table 1 of Stainthorp et al. (2010; though please note that in this Table Year 3 and 4 participants were erroneously denoted as Year 4 and 5).

At the time of the current study, 15 of the original sample of 160 children had moved to other schools. Consent was obtained from parents of all but two of the remaining children (the slight change in sample size hardly affected the means reported in Table 1 of Stainthorp et al., 2010). Therefore, participants in this study were 143 children, 69 in the low RAN group, and 74 Controls. For operational reasons, the children in the two year groups were tested at different times: the older children were in the final term of Year 6 of primary school and the younger children were tested around 3 months later during the first term of Year 6.

\section{Materials and Measures}

RAN (letters and digits) and PA (elision and blending) subtests of the Comprehensive Test of Phonological Processing (CTOPP; Wagner, Torgesen, \& Rashotte, 1999) were administered to ensure that the groups of children still differed on RAN, but were well matched on PA. The British Ability Scales (BAS) single word reading test (Elliot, Murray, \& Pearson, 1983) was also administered as a measure of word reading. 
Orthographic Knowledge and RAN

\section{Exposure to print.}

An Author Recognition Task (ART; Ricketts, Nation, \& Bishop, 2007) was used to assess exposure to print. This requires children to read a checklist of 50 names ( 25 real authors, 25 foils), selecting those they believe to be names of real authors of children's books. Scores were calculated by subtracting the number of foils from the number of real authors identified.

\section{Word reading.}

A pre-publication version of the Diagnostic Test of Word Reading Processes (DTWRP; Forum for Research in Literacy and Language, 2012) was used, which is composed of three sets: 30 words with regular spellings (e.g. 'went'), 30 irregular words (e.g. 'want') and 30 pseudowords (e.g. 'thent'). Letterstrings varied in length from 2-13 letters and from 1-5 syllables. Pseudowords were created through combining components of different regular words (e.g., 'pertue' from 'PERhaps' and 'staTUE'). The sets were well matched on length, bigram frequency, trigram frequency, and 1- and 2-letter different neighbours (derived from the MRC psycholinguistic database; Coltheart, 1981). Regular and irregular word sets were also similar in frequency (Children's Printed Word Database; Masterson, Stuart, Dixon, \& Lovejoy, 2010). The task was computer-administered using E-prime experiment presentation software (Schneider, Eschman, \& Zuccolotto, 2002) on an Apple Macbook laptop running Windows XP, with a 2.2 GHz Intel Core Duo processor and a 13" color screen (as in all the following computer presented tasks). RTs (vocal onset latencies) were recorded using a Psychology Software Tools Serial Response Box voice key. The test was in two parts: first word reading (four practice trials followed by 60 test trials) and then pseudoword reading (four practice trials followed by 30 test trials). On each trial, a fixation 
cross appeared in the centre of the screen for $500 \mathrm{~ms}$, followed by a letterstring, presented in size 26, black 'courier new' font, which remained on screen until participants responded.

\section{Orthographic choice task.}

A computer administered orthographic choice task (Olson, Wise, Conners, Rack, \& Fulker, 1989) was used as a measure of word level orthographic knowledge. Children were required to pick the real word from a correctly spelled/pseudohomophone pair (e.g. rain/rane). Test stimuli were 38 word/pseudohomophone pairs. On each trial a pair of letterstrings appeared on either side of a fixation point, and participants indicated with a keypress whether the real word was on the left or the right of fixation. Accuracy and latency were recorded.

\section{Wordlikeness Task.}

An adapted version of Cassar and Treiman's (1997) wordlikeness task was used, where participants judged which of two pseudowords 'looks more like a real word'. Test stimuli were 20 pairs of mono or bi-syllabic pseudowords (as in Cassar and Treiman's Experiment 1, 1997), each containing a critical letter doublet at the beginning of one, and at the end of the other letterstring (e.g. bbaf; baff). On each trial the pair of letterstrings appeared on either side of a fixation point and remained on screen until children made a keypress response. Accuracy and latency were recorded.

\section{Orthographic learning task.}

An orthographic learning task (adapted from Nation, Angell, \& Castles, 2007) was used. This was a two-phase task involving first exposure and then recall. Exposure involved 
a naming and lexical decision task, where participants first named each letterstring, and then decided whether it was a word or a pseudoword. Unbeknownst to participants, 8 target pseudowords were embedded in this task, with eight words used as fillers for the lexical decision task. Four of the target pseudowords appeared once in the exposure phase, and the remaining four items appeared four times. In the recall phase, participants were asked to try to remember the pseudowords encountered in the earlier exposure phase, and to select each target item (e.g. ferd) from an array of four similar stimuli (e.g. ferd, furd, ferp, furp). The recall phase was administered twice, first in the same test session as the exposure phase following completion of two intervening tasks (immediate recall), and again a week later (delayed recall).

Eight pairs of homophonic pseudowords were used in the study (e.g. goak; goke). One of each pair was the target item in the exposure phase, and the other acted as a homophonic foil in the test phase. The further foils used in the test phase were created by changing the test item's final consonant (e.g. goak; goab).

During the exposure phase, each target stimulus appeared on the screen following the brief presentation of a fixation cross. Participants were asked to read the item aloud as quickly as they could, and then to make a key-press response to indicate whether the letterstring was a word or a pseudoword. There were three practice trials, where feedback was given, followed by 32 test trials. During the recall phase, on each trial four stimuli appeared on the screen, one in each quadrant. Accuracy data were recorded.

\section{Procedure}

The above tasks were separated into two test sessions, each of which comprised larger test batteries lasting around 20 minutes, and administered one week apart. The exposure 
phase of the orthographic learning task was the first task in Session 1, with the first recall phase of the task carried out at the end of this session following other tasks. Session 2 began with the delayed recall phase of the orthographic learning task, and also included wordlikeness and orthographic choice tasks.

\section{Results}

\section{RAN, PA, BAS Single Word Reading, and Print Exposure}

We first sought to establish that the children's profiles were still consistent with their allocation to low RAN and control groups, which occurred at baseline around 3 years prior to the current study. Scores on RAN, PA and BAS single word reading, summarized in Table 1, were subjected to univariate ANOVAs, with RAN group (low RAN, Controls) as a between participant variable, and Age group (younger, older) as a between participant variable in all analyses.

Results showed that the low RAN group remained significantly slower than controls on the RAN task $(F(1,139)=85.78, M S E=190.70, p<.001)$. Neither the main effect of Age group, $F(1,139)=0.53, M S E=190.70, p=.466$, nor the Age group by RAN group interaction, $F(1,139)=1.01, M S E=190.70, p=.317$, were significant. The low RAN group also remained significantly worse on the BAS word reading test than controls, $F(1,139)=$ $10.84, M S E=52.73, p<.001)$; again, neither Age group $F(1,139)=2.07, M S E=52.73, p=$ .153 , nor the Age group by RAN group interaction, $F(1,139)=0.003, M S E=52.73, p=$ .954 , were significant effects. Importantly, the low RAN group were still well matched with controls on PA: neither the effect of RAN Group, $F(1,139)=0.76, M S E=39.95, p=.384$, 
nor of Age group, $F(1,139)=1.11, M S E=39.95, p=.293$, nor the RAN group by Age group interaction, $F(1,139)=1.38, M S E=39.95, p=.242$, were significant.

\section{Exposure to Print}

A RAN group by Age group ANOVA was performed on children's scores on the Author Recognition Test. Data are summarized in Table 1. Analysis revealed that there was no difference between low RAN group and controls on this measure, $F(1,139)=0.08, M S E=$ $8.13, p=.778$, nor was there a significant main effect of Age group, $F(1,139)=1.98, M S E=$ $8.13, p=.162$. The interaction was marginally significant, $F(1,139)=3.83, M S E=8.13, p=$ .053: further analysis revealed that there was a slight difference in favour of the Control group in the younger sample, but in favour of the low RAN group in the older sample, though simple effects tests revealed that neither difference was statistically significant (younger age group: $F(1,67)=1.21, M S E=8.96, p=.275$; older age group: $F(1,72)=2.89, M S E=7.38$, $p=.093$ ). Thus we found no evidence that the low RAN group's reading weakness could be attributed to less experience with print than controls.

Table 1 about here

\section{DTWRP Reading Test}

Accuracy and latency (median RT on correct trials) scores on the DTWRP reading test are presented in Table 1. The means suggest that the low RAN group, both older and younger age groups, were less accurate and slower across all letterstring types. Letterstring type (regular word, irregular word, pseudoword) x RAN group (low RAN, Control) x Age group (younger, older) ANOVAs were carried out, on both accuracy and RT data. Simple 
reaction time (SRT), obtained three years earlier at baseline, was a covariate in analyses involving RT data. To correct a negative skew in accuracy data, it was necessary to apply reflect and logarithm transformation and to exclude seven cases who were univariate outliers on more than one letterstring type. To resolve a significant positive skew in RT data, logarithm transformation was applied and two statistical outliers were excluded.

Accuracy analysis revealed that the low RAN group were significantly less accurate than controls, $F(1,126)=18.39, M S E=0.157, p<.001$. The main effect of Letterstring type was also significant, $F(2,252)=124.71, M S E=0.02, p<.001$, and further analysis revealed that children read irregular words more accurately than regular words, $F(1,129)=25.18$, $M S E=0.02, p<.001$, and that both regular and irregular words were read more accurately than pseudowords (regular: $F(1,129)=286.63, M S E=0.02, p<.001$; irregular: $F(1,129)=$ 88.84, MSE $=0.03, p<.001)$. No other main effects or interactions were significant.

RT analysis showed that the low RAN group was significantly slower than controls to read the letterstrings, $F(1,128)=11.39, M S E=0.02, p=.001$. The main effect of Letterstring type was also significant, $F(2,256)=6.60, M S E=0.004, p=.002$, as was the effect of the covariate (baseline SRT), $F(1,128)=5.74, M S E=0.02, p=.018$. There was also a significant interaction between Letterstring type and RAN group, $F(2,256)=5.39, M S E=$ $0.004, p=.005$. Simple effects tests revealed that the effect of RAN group was significant for regular and irregular words and pseudowords, with the interaction seemingly driven by between group differences that were greater for pseudowords than for either regular or irregular words.

While performance on the DTWRP reading test (both accuracy and latency) provided evidence of the low RAN group's overall lower reading ability, we next addressed the degree to which this group difference reflected specific lexical orthographic difficulties, over and 
above decoding difficulties. Adding DTWRP pseudoword reading scores (as the measure of decoding) as a covariate to ANCOVA was precluded by the significant difference between groups on this variable (see Miller \& Chapman, 2001, for a discussion of this issue). We therefore carried out hierarchical regression analyses on both accuracy and RT data to examine whether RAN group, encoded as a dummy variable, remained a predictor of DTWRP irregular word reading, after first controlling for DTWRP pseudoword reading, which was entered at the previous step. Baseline SRT was entered as an additional variable at Step 1 in the RT analysis. To correct a significant departure from normality in the distribution of residuals in the accuracy analysis, it was necessary to remove four multivariate outliers. In the RT analysis, it was also necessary to exclude four multivariate outliers to normalize residuals. Age group was excluded as it did not correlate with reading scores (see Table 3).

Table 2 about here

Results of regressions are shown in Table 2, which shows change in $R^{2}$ (with significance level) after each step, standardized betas (with significance levels) for each variable as entered at the final step, and total $R^{2}$. RAN group remained a significant predictor of irregular word reading after accounting for pseudoword reading scores, in both accuracy and RT analyses, giving support to our prediction of an orthographic locus of the low RAN group's reading difficulty.

\section{Orthographic Choice Task}

Children's accuracy and median RTs (on correct trials) on the orthographic Choice are summarized in Table 1 and were subjected to ANOVAs, with RAN group and Age group as between participant factors. Baseline SRT was a covariate in the RT analysis. Logarithm 
transformation was applied to the latency data and two statistical outliers were removed from the dataset to correct a positive skew.

Analysis revealed that the low RAN group was significantly less accurate and slower than controls (accuracy: $F(1,137)=7.12, M S E=0.01, p=.009$; RTs: $F(1,134)=6.40, M S E$ $=0.01, p=.013$. The main effect of Age group was significant in the accuracy but not the RT analysis (Accuracy: $F(1,137)=8.53, M S E=0.01, p=.004 ; \mathrm{RT}: F(1,134)=0.394, M S E$ $=0.01, p=.531)$. Neither accuracy nor RT analysis revealed a significant interaction between RAN group and Age group (accuracy: $F(1,137)=1.24, M S E=0.01, p=.268$; RT: $F(1,134)=0.39, M S E=0.01, p=.531)$. SRT was not a significant covariate in the RT analysis, $F(1,134)=2.07, M S E=0.01, p=.152$.

We next investigated whether the Low RAN group's performance on the orthographic choice task reflected a specific problem with orthography, over an above their relatively poor reading, using hierarchical regression analysis. In the accuracy analysis, RAN group was encoded as a dummy variable and entered last into the regression, after DTWRP pseudoword reading accuracy (as a measure of decoding ability) and Age group. In the RT analysis, RAN group was entered at Step 2 after DTWRP pseudoword RT score and SRT at Step 1 (age group was excluded given its non-significant correlation with orthographic choice). To normalize the distribution of residuals in the RT data, it was necessary to remove 3 multivariate outliers.

As shown in Table 2, while DTWRP pseudoword reading accuracy and Age group each accounted for significant variance in orthographic choice accuracy when entered at step 1, the inclusion of the RAN group dummy variable at the final step did not significantly increase $R^{2}$. A similar pattern emerged in the RT analysis: DTWRP reading RTs (but not SRT) accounted for unique variance in orthographic choice latencies but RAN group, entered 
at the final step, did not account for additional variance. Thus the orthographic choice task provided us with no additional evidence of a specific deficit in word level orthographic knowledge in the low RAN group.

Table 3 about here

\section{Wordlikeness Task}

The wordlikeness task can be seen as a measure of orthographic pattern knowledge at the sub-word level. As this task was originally designed for younger children, accuracy was close to ceiling so we analyzed only latency data (median RTs on correct trials). Data were significantly positively skewed, and to correct this we applied logarithm transformation, and excluded four univariate outliers. Data were subjected to ANCOVA, with RAN group and Age group as between participant factors, and baseline SRT as the covariate. The low RAN group was significantly slower than controls on the wordlikeness task, $F(1,127)=8.18, M S E$ $=0.01, p=.005$. Baseline SRT was a significant covariate, $F(1,127)=4.01, M S E=0.01, p$ $=.047$, though neither the effect of Age group, $F(1,127)=0.22, M S E=0.01, p=.641$, nor the RAN group by Age group interaction, $F(1,127)=0.60, M S E=0.01, p=.40$, reached significance.

We next subjected wordlikeness data to hierarchical regression analysis, though it was first necessary to remove a further four multivariate outliers from the dataset to normalize the residuals' distribution. Age group did not correlate with wordlikeness, so was excluded.

Table 2 shows that at step 1, DTWRP pseudoword reading, but not baseline SRT, accounted for unique variance in wordlikeness RTs. However, when entered at the last step, RAN group accounted for a small but significant amount of additional variance in wordlikeness RTs. These results suggest that the low RAN group may have a specific difficulty with orthographic pattern learning. 


\section{Orthographic Learning Task}

Finally, we examined performance on the orthographic learning task, a test of ability to form new, word-level orthographic representations. Table 4 shows mean (and SD) proportion of correct trials, in both immediate and delayed recall conditions and for both single and four exposure conditions. Recall data were subjected to ANOVA, with RAN group and Age group as between participant factors, and Exposure (single, four) and Test Time (immediate, delayed) as within participant factors. Surprisingly, results indicated that the low RAN group was significantly more accurate on this task than controls, $F(1,137)=3.98, M S E$ $=0.10, p=.048$. Participants were more accurate after four than one exposures $(F(1,137)=$ 125.26, $M S E=0.09, p<.001$ and in the immediate than delayed recall condition $(F(1,137)=$ $15.53, M S E=0.04, p<.001)$. The RAN group by Year group by Test time by Exposure interaction approached significance, $F(1,137)=3.67, M S E=0.04, p=.058$, but no other main effects or interactions were significant (Exposure by RAN group: $F(1,137)=1.27$, $M S E=0.09, p=.262$. All other $F \mathrm{~s}<1)$.

Table 4 about here

\section{Discussion}

The first aim of the current research was to investigate the persistence of a RAN deficit and associated word reading deficit, in English children. Results showed that nearly three years after they were first identified, children in the low RAN group remained significantly slower on RAN and were worse word readers than controls. Indeed, contrary to 
findings of correlational, longitudinal research (Torgesen et al., 1997; Wagner et al., 1997), the severity of the low RAN group's word reading deficit had not diminished over time, despite the groups being still well matched on PA. This is consistent with other reports of the enduring nature of RAN-related reading difficulties (Kirby et al., 2003; Landerl \& Wimmer, 2008).

The second main aim of the current research was to address a debate in the literature concerning the degree to which RAN difficulties are associated, perhaps causally, with deficits in orthographic knowledge. Initial analysis of word reading, orthographic choice, and wordlikeness task data revealed that the low RAN group were consistently outperformed by the control group. However, as discussed in the introduction, before we took this as evidence of orthographic deficits linked to RAN problems, it was first necessary to take two factors into account. First, Cunningham and Stanovich (1993) showed that print exposure predicts unique variance in orthographic knowledge, raising the possibility that any orthographic weakness may be due not to a RAN deficit per se, but rather to less experience with print. Second, decoding ability is intricately linked to orthographic knowledge. According to Share (1995), phonological recoding is the mechanism for orthographic learning, and furthermore, all of the tasks we used to assess orthographic knowledge require decoding skill. We therefore wished to establish the degree to which any orthographic difficulty shown by the RAN group was not merely a reflection of poor decoding abilities.

With regard to the role of print exposure, analysis of the children's performance on the Author Recognition Task provided no evidence of a difference between the low RAN group and controls. This is interesting as print exposure has not typically been assessed alongside RAN in research of this kind, and somewhat surprising given that reading is slower 
and presumably more effortful for children with RAN difficulties. The finding suggests that any orthographic deficit in the RAN group was not simply a result of less reading experience.

Initial analysis of performance on the DTWRP reading test confirmed the Low RAN group's relative reading impairment, though their deficit on irregular words was no greater than on regular words and pseudowords. However, hierarchical regression analyses revealed that RAN group predicted unique variance in both accuracy and latency, after controlling for decoding ability and thus provided some evidence of a specific deficit in orthographic knowledge.

A different pattern emerged when we considered orthographic choice task performance. Regression analysis revealed that the between group difference on this task no longer held, after controlling for decoding ability, suggesting that the low RAN group's disadvantage was more a function of general reading ability than of orthographic knowledge. This finding is not consistent with Georgiou et al. (2009), but this may reflect the fact that decoding ability was not controlled in their study.

We next investigated orthographic knowledge at the level of sub-word level orthographic patterns using the wordlikeness task. In this case, RAN group accounted for a small but significant amount of variance in wordlikeness performance, even after controlling for decoding ability. This is contrary to findings reported by Georgiou et al. (2009), who found no association between RAN and performance on a wordlikeness task, even without controlling for reading ability, though this may have been due to the ceiling effect they reported. However, our findings are consistent with those of Bowers et al. (1999), who reported a deficit in orthographic pattern learning in their RAN deficit group. Bowers et al. (1999) interpreted their finding as supportive of the mechanistic account of the RAN-reading 
association provided by Double Deficit theory (e.g. Wolf \& Bowers, 1999), which predicts a specific difficulty in learning commonly occurring letter clusters.

Previous research (Moll et al., 2009; van den Boer et al., 2013) has shown, like ours, that RAN was as strongly related to pseudoword as to word reading. Moll et al. also found that RAN was less strongly linked than PA to inconsistent word spelling, which they took as a measure of whole word orthographic knowledge. They interpreted their findings as evidence against the view that RAN deficits are associated with orthographic deficits, arguing instead that RAN deficits impact on reading through impairing the automaticity of mappings between orthography and phonology at the level of letters and letter clusters. Whether the root of this apparent deficit in orthographic pattern learning lies in the quality of the representations themselves, or in the mappings between orthographic and phonological representations, is an important empirical question which cannot be answered by Moll et al.'s (2009) data or our own.

While conceding that pseudoword reading fluency may also be influenced by orthographic knowledge, Moll et al. (2009) argued that RAN's stronger relation to pseudoword than to word reading fluency must speak against an association between RAN and orthographic knowledge because "words would typically include a higher number of typical orthographic patterns than nonwords" (Moll et al., 2009, p. 19). This is not necessarily the case, in English at least. In the DTWRP there are no differences between regular, irregular and pseudoword sub-word level lexical characteristics.

Given that the low RAN group were not disadvantaged on irregular words, but showed evidence of deficits in sub-word level orthographic patterns, it may be that lexical consistency is the critical factor in understanding the link between RAN and reading. Findings are perhaps most in line with theories of reading that do not posit a sharp distinction 
between different levels of lexical, orthographic knowledge and where representations of words are distributed, rather than local. According to the Triangle Model of reading (e.g. Plaut, McClelland, Seidenberg, \& Patterson, 1996) words are represented as distributed patterns of activity across different pools of units representing orthography, phonology and semantics, with a componential relationship between orthography and phonology emerging through learning. Further research systematically manipulating consistency may determine whether children with RAN deficits are less sensitive to consistency than controls, perhaps as a result of difficulties learning orthographic patterns.

Our most surprising finding was the low RAN group's apparent strength on the orthographic learning task, despite their weakness in orthographic knowledge. This could simply be because reading is more effortful for the low RAN group, requiring more attention as decoding is less automatized, resulting in greater depth of processing during the exposure phase, and thus greater recall. However, one would then expect a negative relationship between reading ability and performance on the orthographic learning task, which was not obtained. An alternative explanation might be that children with RAN deficits could compensate for their reading difficulties by adopting different strategies, for example relying more on visual memory to store orthographic knowledge. Because the stimuli we used were all relatively simple and short, it could be that a visual memory strategy may have been effective, though it is not likely to be an optimal strategy for learning more generally, given that most words in English are longer and more complex. This raises an empirical question, which would be resolved through an examination of visual memory in children with RAN deficits, and through a further test of orthographic learning with more complex, longer stimuli.

\section{Conclusion}


This study provided evidence of the enduring nature of RAN difficulties and the reading difficulties that accompany them, even in children with age-appropriate PA. Furthermore, there was evidence of a deficit in both word and sub-word level orthographic knowledge in the low RAN group, at an age where, with transition to secondary school, independent reading skills become crucial for success across the curriculum, and where orthographic processes should come to dominate children's maturing reading systems. Conclusions are somewhat limited by the null finding on the orthographic choice task, and by only having a single measure of sub-word orthographic pattern knowledge. On the other hand, the fact that low RAN and control groups were equated for both PA and print exposure, two important potential confounds, lends weight to this interpretation, and adds to research in this field which has not assessed print exposure. These findings are consistent with the Double Deficit view of dyslexia, and are perhaps most easily accommodated within a theoretical framework such as the Triangle model, where orthographic knowledge at different levels emerges with reading experience. Our surprising finding of an advantage on the orthographic learning task in the low RAN group suggests that future work must investigate the strategies adopted in orthographic learning in children with this profile.

\section{Acknowledgements}

The research described in this paper was supported by ESRC grant numbers RES000-23-0699 and RES-000-22-2387. We thank the participants and their teachers for their long-term cooperation with this research. 
Orthographic knowledge and RAN

\section{References}

Apel, K. (2011). What Is Orthographic Knowledge? Language, Speech, and Hearing Services in Schools, 42, 592-603. doi:10.1044/0161-1461(2011/10-0085)a

Badian, N. A. (1993). Phonemic awareness, naming, visual symbol processing, and reading. Reading and Writing, 5(1), 87-100. doi:10.1007/BF01026920

Bosse, M.-L., Tainturier, M. J., \& Valdois, S. (2007). Developmental dyslexia: the visual attention span deficit hypothesis. Cognition, 104(2), 198-230. doi:10.1016/j.cognition.2006.05.009

Bowers, P. G., Sunseth, K., \& Golden, J. (1999). The Route Between Rapid Naming and Reading Progress. Scientific Studies of Reading, 3(1), 37-53.

Bowers, P. G., \& Wolf, M. (1993). Theoretical links among naming speed, precise timing mechanisms and orthographic skill in dyslexia. Reading and Writing, 5(1), 69-85. doi:10.1007/BF01026919

Bowey, J. A., \& Miller, R. (2007). Correlates of orthographic learning in third-grade children's silent reading. Journal of Research in Reading, 30(2), 115-128. doi:10.1111/j.1467-9817.2007.00335.x

Bowey, J. A., \& Muller, D. (2005). Phonological recoding and rapid orthographic learning in third-graders' silent reading: a critical test of the self-teaching hypothesis. Journal of Experimental Child Psychology, 92(3), 203-19. doi:10.1016/j.jecp.2005.06.005

Cassar, M., \& Treiman, R. (1997). The beginnings of orthographic knowledge: Children’s knowledge of double letters in words. Journal of Educational Psychology, 89, 631-644. 
Orthographic knowledge and RAN

Castles, A., \& Nation, K. (2006). How does orthographic learning happen? In S. Andrews (Ed.), From inkmarks to ideas: Current issues in lexical processing (pp. 151-179). Hove, UK: Psychology Press.

Coltheart, M. (1981). The MRC psycholinguistic database. The Quarterly Journal of Experimental Psychology Section A, 33(4), 497-505. doi:10.1080/14640748108400805

Conrad, N. J., \& Levy, B. A. (2006). Letter Processing and the Formation of Memory Representations in Children with Naming Speed Deficits. Reading and Writing, 20(3), 201-223. doi:10.1007/s11145-006-9028-8

Cunningham, A. E., \& Stanovich, K. E. (1993). Children's literacy environments and early word recognition subskills. Reading and Writing, 5(2), 193-204. doi:10.1007/BF01027484

Denckla, M. B., \& Rudel, R. (1974). Rapid “automatized” naming of pictured objects, colors, letters and numbers by normal children. Cortex: A Journal Devoted to the Study of the Nervous System and Behavior, 10(2).

Ehri, L. C. (2005). Learning to Read Words: Theory, Findings, and Issues. Scientific Studies of Reading, 9(2), 167-188. doi:10.1207/s1532799xssr0902_4

Elliot, C. D., Murray, D. J., \& Pearson, L. S. (1983). British Ability Scales. Windsor UK: NFERNelson.

Forum for Research in Literacy and Language, Institute of Education (2012). The Diagnostic Test of Word Reading Processes. Swindon, UK: G L Assessment.

Georgiou, G. K., Parrila, R., Cui, Y., \& Papadopoulos, T. C. (2013). Why is rapid automatized naming related to reading? Journal of experimental child psychology, 115(1), 218-25. doi:10.1016/j.jecp.2012.10.015 
Orthographic knowledge and RAN

Georgiou, G. K., Parrila, R., \& Kirby, J. R. (2009). RAN Components and Reading Development From Grade 3 to Grade 5: What Underlies Their Relationship? Scientific Studies of Reading, 13(6), 508534. doi:10.1080/10888430903034796

Georgiou, G. K., Torppa, M., Manolitsis, G., Lyytinen, H., \& Parrila, R. (2010). Longitudinal predictors of reading and spelling across languages varying in orthographic consistency. Reading and Writing, 25(2), 321-346. doi:10.1007/s11145-010-9271-x

Kail, R., Hall, L. K., \& Caskey, B. J. (1999). Processing speed, exposure to print, and naming speed. Applied Psycholinguistics, 20(02), 303-314.

Kirby, J. R., Parrila, R. K., \& Pfeiffer, S. L. (2003). Naming speed and phonological awareness as predictors of reading development. Journal of Educational Psychology, 95(3), 453-464. doi:10.1037/0022-0663.95.3.453

Landerl, K., \& Wimmer, H. (2008). Development of word reading fluency and spelling in a consistent orthography: An 8-year follow-up. Journal of Educational Psychology, 100(1), 150-161. doi:10.1037/0022-0663.100.1.150

Manis, F., Seidenberg, M., \& Doi, L. M. (1999). See Dick RAN: Rapid naming and the longitudinal prediction of reading subskills in first and second graders. Scientific Studies of Reading, 3(2), $129-157$.

Miller, G., \& Chapman, J. (2001). Misunderstanding analysis of covariance. Journal of Abnormal Psychology, 110(1), 40-48. 
Orthographic knowledge and RAN

Moll, K., Fussenegger, B., Willburger, E., \& Landerl, K. (2009). RAN Is Not a Measure of Orthographic Processing. Evidence From the Asymmetric German Orthography. Scientific Studies of Reading, 13(1), 1-25. doi:10.1080/10888430802631684

Nation, K., Angell, P., \& Castles, A. (2007). Orthographic learning via self-teaching in children learning to read English: effects of exposure, durability, and context. Journal of Experimental Child Psychology, 96(1), 71-84. doi:10.1016/j.jecp.2006.06.004

Norton, E. S., \& Wolf, M. (2011). Rapid Automatized Naming (RAN) and Reading Fluency: Implications for Understanding and Treatment of Reading Disabilities. Annual Review of Psychology, 63, 14-41. doi:10.1146/annurev-psych-120710-100431

Olson, R., Wise, B., Conners, F., Rack, J., \& Fulker, D. (1989). Specific deficits in component reading and spelling skills: Genetic and environmental influences. Journal of Learning Disabilities, 22, $339-348$.

Plaut, D. C., McClelland, J. L., Seidenberg, M. S., \& Patterson, K. (1996). Understanding normal and impaired word reading: computational principles in quasi-regular domains. Psychological Review, 103(1), 56-115.

Powell, D., Stainthorp, R., Stuart, M., Quinlan, P., \& Garwood, H. (2007). An experimental comparison between rival theories of Rapid Automatized Naming (RAN) performance and its relationship to reading. Journal of Experimental Child Psychology, 98(1), 46-68. doi: 10.1016/j.jecp.2007.04.003

Powell, D., Stainthorp, R. \& Stuart, M. (2008) A deficit in orthographic knowledge, but not orthographic learning, in children poor at Rapid Automatized Naming tasks. Paper presented at the Annual Meeting of the Society for the Scientific Study of Reading, Asheville, NC, USA. 
Orthographic knowledge and RAN

Protopapas, A., Altani, A., \& Georgiou, G. K. (2013). RAN Backward: A Test of the Visual Scanning Hypothesis. Scientific Studies of Reading, (June), 1-9. doi:10.1080/10888438.2013.769556

Ricketts, J., Nation, K., \& Bishop, D. V. M. (2007). Vocabulary is important for some, but not all reading skills. Scientific Studies of Reading, 11(3), 235-257.

Schneider, W., Eschman, A., \& Zuccolotto, A. (2002). E-Prime reference guide. Pittsburgh: Psychology Software Tools, Inc.

Share, D. L. (1995). Phonological recoding and self-teaching: sine qua non of reading acquisition. Cognition, 55(2), 151-218. doi:10.1016/0010-0277(94)00645-2

Stainthorp, R., Powell, D., \& Stuart, M. (2013). The relationship between rapid naming and word spelling in English. Journal of Research in Reading, 36(4), 371-388. doi: 10.1111/jrir.12002

Stainthorp, R. W., Powell, D., Stuart, M., Quinlan, P., \& Garwood, H. (2010) Visual processing deficits in children with slow RAN performance. Scientific Studies of Reading, 14 (3), 266-292.

Torgesen, J., Wagner, R., Rashotte, C. A., Burgess, S. R., \& Hecht, S. (1997). contributions of phonological awareness and rapid automatic naming ability to the growth of word-reading skills in second- to fifth-grade children. Scientific Studies of Reading, 1(2), 161-185.

van den Boer, M., de Jong, P. F., \& Haentjens-van Meeteren, M. M. (2013). Modeling the Length Effect: Specifying the Relation With Visual and Phonological Correlates of Reading. Scientific Studies of Reading, 17(4), 243-256. doi:10.1080/10888438.2012.683222 
Orthographic knowledge and RAN

Vellutino, F. R., Fletcher, J. M., Snowling, M. J., \& Scanlon, D. M. (2004). Specific reading disability (dyslexia): what have we learned in the past four decades? Journal of Child Psychology and Psychiatry, and Allied Disciplines, 45(1), 2-40.

Wagner, R. K., W, J. K., Rashotte, C. A, Hecht, S. A, Barker, T. A, Burgess, S. R., Donahue, J., et al. (1997). Changing relations between phonological processing abilities and word-level reading as children develop from beginning to skilled readers: a 5-year longitudinal study. Developmental Psychology, 33(3), 468-79.

Wagner, R. K., Torgesen, J. K., \& Rashotte, C. A. (1999). Comprehensive Test of Phonological Processing. Austin, TX: PRO-ED.

Wechsler, D. (1992). Wechsler Intelligence Scale for Children. $3^{\text {rd }}$. Edition. New York: Psychological Corporation.

Wolf, M., \& Bowers, P. G. (1999). The double-deficit hypothesis for the developmental dyslexias. Journal of Educational Psychology, 91(3), 415. 
Table 1: Summary of scores on PA, RAN, BAS single word reading, Author Recognition task (ART), orthographic choice, wordlikeness and orthographic learning tasks.

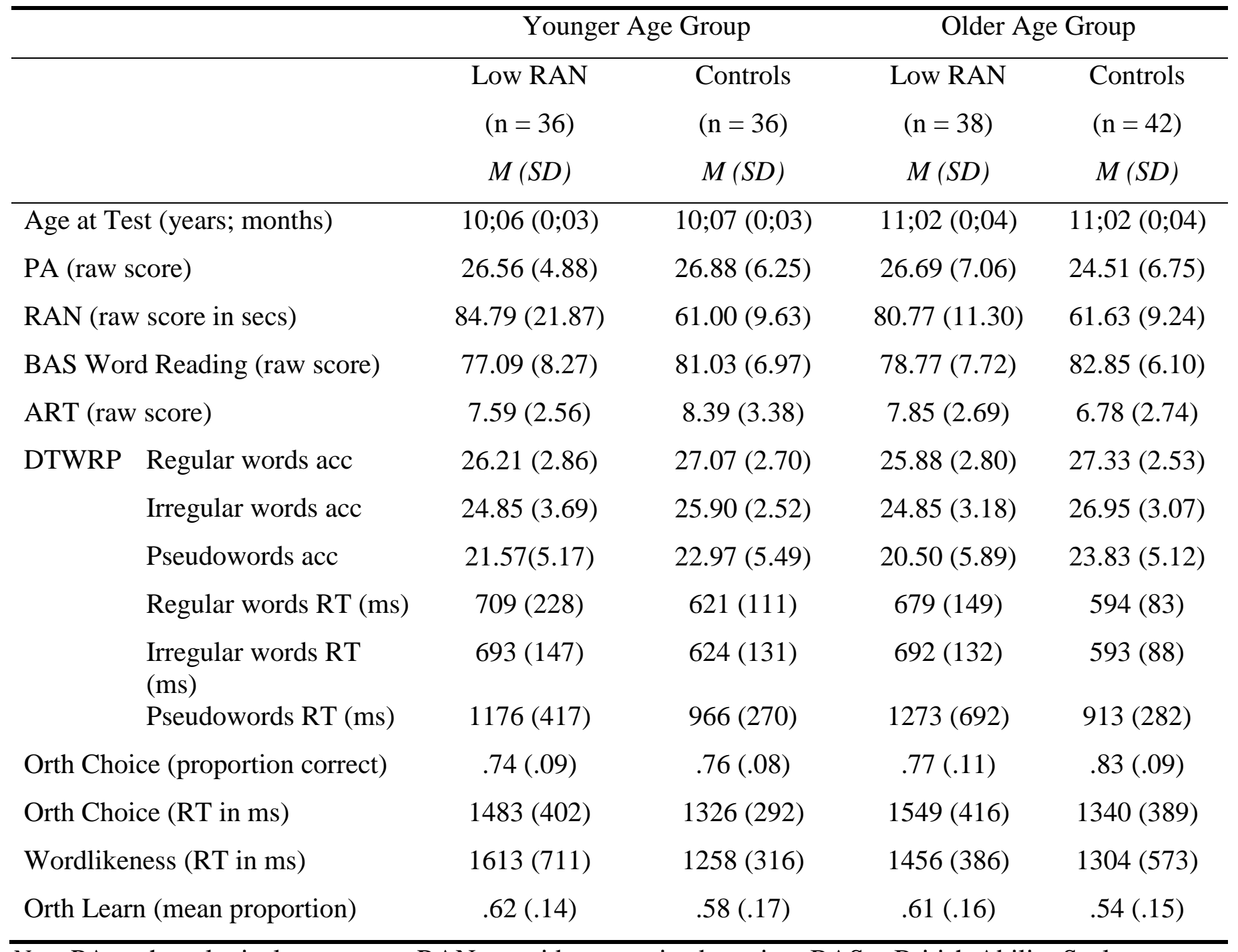

Note PA = phonological awareness; RAN = rapid automatized naming; BAS = British Ability Scales; ART $=$ Author Recognition Task; DTWRP $=$ Diagnostic Test of Word Reading Processes; acc $=$ accuracy; Orth Choice $=$ Orthographic Choice; Orth Learn $=$ Orthographic Learning task (mean proportion across all conditions). 
Table 2: Hierarchical regression analyses of a) DTWRP irregular word reading accuracy scores (left) and RTs (right) and b) Orthographic choice accuracy scores and RTs, and c) wordlikeness RTs.

\begin{tabular}{|c|c|c|c|c|c|}
\hline \multirow[b]{2}{*}{ Step } & \multirow[b]{2}{*}{ Variable } & \multicolumn{2}{|c|}{ Accuracy analysis } & \multicolumn{2}{|c|}{ RT analysis } \\
\hline & & $\Delta R^{2}$ & $\beta$ & $\Delta R^{2}$ & $\beta$ \\
\hline \multicolumn{6}{|c|}{ a) DTWRP Irregular word reading } \\
\hline \multirow[t]{2}{*}{1} & SRT & & & & .06 \\
\hline & DTWRP pseudoword reading & $.50 * * *$ & $.66 * * *$ & $.53 * * *$ & $.67 * * *$ \\
\hline 2 & RAN Group (dummy) & $.02 *$ & $-.15^{*}$ & $.02 *$ & $.14^{*}$ \\
\hline \multicolumn{2}{|c|}{ Total $R^{2}$} & $.52 * * *$ & & $.55^{* * *}$ & \\
\hline \multicolumn{6}{|c|}{ b) Orthographic choice } \\
\hline \multirow[t]{3}{*}{1} & Age group (dummy) & & $.23 * *$ & & \\
\hline & SRT & & & & .02 \\
\hline & DTWRP pseudoword reading & $.28 * * *$ & $.44 * * *$ & $.28 * * *$ & $.50 * * *$ \\
\hline 2 & RAN group (dummy) & .01 & -.12 & .01 & .07 \\
\hline \multicolumn{2}{|c|}{ Total $R^{2}$} & $.29 * * *$ & & $.29 * * *$ & \\
\hline \multicolumn{6}{|c|}{ c) Wordlikeness } \\
\hline \multirow[t]{3}{*}{1.} & Age group (dummy) & & & & \\
\hline & SRT & & & & .15 \\
\hline & DTWRP pseudoword reading & & & $.14 * * *$ & $.24 * *$ \\
\hline 2. & RAN group (dummy) & & & $.03 *$ & $.19 *$ \\
\hline \multicolumn{2}{|c|}{ Total $R^{2}$} & & & $.17 * * *$ & \\
\hline
\end{tabular}

$* p<.05 ; * * p<.01 ; * * * p<.001$. NB. SRT = simple reaction time (obtained at baseline, three years previously); RAN = rapid automatized naming; DTWRP = Diagnostic Test of Word Reading Processes 
Table 3. Pearson's correlations between the dummy variable encoding RAN Group, Age group and children's scores on simple reaction time,

BAS word reading (accuracy), DTWRP reading (individual subtests accuracy and RT data; mean accuracy and RT data; orthographic choice (accuracy and RT), wordlikeness (RT), and orthographic learning tasks (grand mean accuracy, across all conditions).

\begin{tabular}{|c|c|c|c|c|c|c|c|c|c|c|c|c|c|}
\hline 2 & 3 & 4 & 5 & 6 & 7 & 8 & 9 & 10 & 11 & 12 & 13 & 14 & 15 \\
\hline 1. RAN group & -.15 & $.27 * *$ & -.04 & $.26 * *$ & $.26 * *$ & $.25 * *$ & $-.30 * *$ & $-.35 * *$ & $-.33 * *$ & $.23 * *$ & $-.24 * *$ & $-.27 * *$ & $-.18 *$ \\
\hline 2. Age group & $-.22 * *$ & .13 & -.12 & -.01 & .13 & .03 & -.11 & -.08 & -.05 & $.25 * *$ & .03 & -.06 & -.08 \\
\hline 3. SRT & & -.10 & $.20 *$ & -.01 & -.13 & -.02 & $.25 * *$ & $.23 * *$ & $.21 *$ & $-.18 *$ & .15 & .16 & .05 \\
\hline 4. BAS reading & & & $.38 * *$ & $.75 * *$ & $.79 * *$ & $.70 * *$ & $-.50 * *$ & $-.50 * *$ & $-.46 * *$ & $.54 * *$ & $-.30 * *$ & -.14 & -.01 \\
\hline 5. ART & & & & $.36 * *$ & $.33 * *$ & $.31 * *$ & $-.20 *$ & $-.21 *$ & $-.19 *$ & $.22 * *$ & -.15 & .02 & $.16 \dagger$ \\
\hline 6. DTWRP Regular Acc & & & & & $.75^{* *}$ & $.78 * *$ & $-.42 * *$ & $-.42 * *$ & $-.39 * *$ & $.47 * *$ & $-.36 * *$ & $-.19 *$ & .04 \\
\hline 7. DTWRP Irregular Acc & & & & & & $.65^{* *}$ & $-.53 * *$ & $-.52 * *$ & $-.50 * *$ & $.53 * *$ & $-.38 * *$ & -.10 & .03 \\
\hline 8. DTWRP Pseudoword Acc & & & & & & & $-.39 * *$ & $-.37 * *$ & $-.32 * *$ & $.47 * *$ & $-.32 * *$ & $-.20 *$ & .04 \\
\hline 9. DTWRP Regular RT & & & & & & & & $.88 * *$ & $.67 * *$ & $-.38 * *$ & $.49 * *$ & $.35^{* *}$ & .08 \\
\hline 10. DTWRP Irregular RT & & & & & & & & & $.71 * *$ & $-.36 * *$ & $.46^{* *}$ & $.36 * *$ & .08 \\
\hline 11. DTWRP Pseudoword RT & & & & & & & & & & $-.34 * *$ & $.53 * *$ & $.33 * *$ & .12 \\
\hline 12. OC Acc & & & & & & & & & & & $-.30 * *$ & $-.20 *$ & -.08 \\
\hline 13. OC RT & & & & & & & & & & & & $.55^{* *}$ & .02 \\
\hline 14. Wordlike RT & & & & & & & & & & & & & .09 \\
\hline 15. Orth Learn & & & & & & & & & & & & & - \\
\hline
\end{tabular}

$\dagger<.07 ; * \mathrm{p}<.05 ; * * \mathrm{p}<.01 ; * * * \mathrm{p}<.001$.

Note SRT = Simple Reaction Time task (obtained at baseline, three years previously); BAS = British Ability Scales Single Word Reading test, raw score; ART = Author Recognition Task; DTWRP = Diagnostic Test of Word Reading Processes; Acc $=$ accuracy score; RT = mean latency on correct trials; OC $=$ Orthographic Choice task; Wordlike $=$ Wordlikeness task; Orth Learn= Orthographic Learning (grand mean across all conditions) 
Table 4: Performance on orthographic Learning Task (proportion correct), for both low RAN group and controls, and both younger and older age groups.

\begin{tabular}{lcccc}
\hline & \multicolumn{2}{c}{ Younger Age group } & \multicolumn{2}{c}{ Older Age group } \\
\cline { 2 - 5 } & \multicolumn{2}{c}{ Low RAN } & Controls & \multicolumn{2}{c}{ Low RAN } & Controls \\
& $M(S D)$ & $M(S D)$ & $M(S D)$ & $M(S D)$ \\
\cline { 2 - 5 } Single exposure & \multicolumn{2}{c}{ Immediate Recall } & \multicolumn{2}{c}{ Immediate Recall } \\
Four exposures & $.54(.25)$ & $.44(.28)$ & $.50(.28)$ & $.44(.26)$ \\
& $.76(.22)$ & $.77(.24)$ & $.75(.26)$ & $.74(.23)$ \\
Single exposure & \multicolumn{2}{c}{ Delayed Recall } & \multicolumn{2}{c}{ Delayed Recall } \\
Four exposures & $.42(.27)$ & $.40(.29)$ & $.49(.25)$ & $.33(.25)$ \\
Mean & $.76(.24)$ & $.69(.24)$ & $.69(.25)$ & $.66(.27)$ \\
\hline
\end{tabular}

